\section{Cefalea crónica diaria en niños y adolescentes}

La cefalea crónica diaria (CCD) es una entidad clínica definida por la presencia frecuente de cefaleas (más de 15 días al mes, en un período de, por lo menos, tres meses).

A partir de los estudios epidemiológicos se confirma que la CCD es poco frecuente en la población adulta (prevalencia del 4,7\%) [1] y aparentemente rara en niños y adolescentes (prevalencia del 0,2-0,9\%) [2,3].

La clasificación de la International Headache Society (IHS) de 1988 trataba el diagnóstico de las cefaleas de acuerdo con las características clínicas de un episodio de cefalea, sin considerar elementos de su historia natural. Como la cefalea de tensión crónica (CTC) era la única categoría de CCD, muchos pacientes quedaban sin clasificar. Entre éstos, la mayoría tenía frecuentemente una historia previa de migraña episódica que había sufrido un período de 'transformación', con crisis progresivamente más frecuentes y una concomitante disminución de la intensidad y pulsatilidad del dolor y la atenuación de los síntomas de náusea, fonofobia y fotofobia.

La clasificación propuesta y seguidamente revisada por Silberstein et al [4] y parcialmente incorporada en la clasificación de la IHS de 2004, agrupa la CCD en cuatro categorías: migraña crónica (MC), CTC, cefalea persistente diaria de novo (CPDN) y hemicraneal continua (HC).

En un estudio de Silberstein et al [4] de 150 adultos con CCD, el $15 \%$ se clasificaron como CTC y el $78 \%$ como MC -no se identificaron en este grupo, ni CPDN ni HC-. La bibliografía en este tema considera unánimemente la CPDN y la $\mathrm{HC}$ como raras en adultos.

La bibliografía sobre la CCD en niños y adolescentes es escasa, y la clasificación de los pacientes se realizó con criterios que variaban en el tiempo, teniendo en cuenta la evolución de la clasificación de las cefaleas crónicas en los adultos.

Del análisis de los estudios realizados en niños y adolescentes con CCD (Tabla I), se comprueba que los resultados son dispares, debido fundamentalmente a los diferentes criterios de clasificación [5-11].

Generalmente, en los estudios más recientes, en los que se utilizó como modelo la clasificación propuesta por Silberstein, predomina claramente el diagnóstico de MC. El estudio de Abu-Arafeh [6], que utilizaba la clasificación de la IHS de 1988 , identificó un $63,8 \%$ de los pacientes con cefalea tensional, pero más del $50 \%$ presentaban náuseas, fonofobia y fotofobia, síntomas claramente migrañosos.

Otro factor de disparidad es la inclusión por parte de algunos autores de una categoría de diagnóstico de la cefalea comórbida o mixta, que reúne a pacientes con cefaleas tensionales y migraña.

Constituye un dato interesante que la categoría CPDN es claramente más frecuente en niños que en la población adulta en los trabajos de Koenig et al [8], Gladstein et al [5] y Moore et al [10]. Las infecciones (principalmente de Salmonella, Escherichia coli, Streptococcus y virus de Epstein-Barr) [5-7] pueden ser un hecho desencadenante de la CPDN y son claramente más frecuentes en este grupo de edad.

No está claro si el mecanismo responsable del establecimiento y perpetuación de una cefalea crónica es común a todos los tipos de cefalea, pero la considerable desproporción entre CPDN y otros tipos de CCD parece argumentar a favor de que esta situación puede ser excepcional.

En nuestro trabajo intentamos identificar los tipos de cefalea crónica encontrada en una consulta de neurología pediátrica, y consideramos aspectos específicos de las cefaleas en este grupo de edad (menor capacidad de explicar claramente el tipo de dolor y su localización e intensidad).

Revisamos los procesos de 397 pacientes de la consulta de Neurología del Hospital de Dona Estefânia con diagnóstico de cefalea, controlados durante el período comprendido entre junio de 2001 y junio de 2003, e identificamos los pacientes que cumplían los criterios de CCD.

Todos los datos referentes a cada paciente se obtuvieron a partir de la consulta de los procesos y se confirmaron, si era necesario, a través de la entrevista telefónica con los pa- dres y con el propio paciente: edad y sexo, historia familiar de cefaleas, historia anterior con inclusión de historia previa de cefalea episódica, progresión de cefalea episódica hacia cefalea crónica, uso de analgésicos, tipo, intensidad y localización de la cefalea, presencia de náuseas, vómitos, fonofobia y fotofobia, interrupción o inhibición de la actividad.

Se excluyeron todos los pacientes identificados con otra patología neurológica o patología sistémica coexistente que pudiera asociarse con cefaleas.

Del total de los 397 niños con diagnóstico de cefaleas, $27(6,25 \%)$ cumplían los criterios de CCD. De ellos, 8 eran de sexo masculino $(29,6 \%)$ y 19 del femenino $(69,4 \%)$. Las edades en el momento del estudio estaban comprendidas entre los 6 y los 15 años (edad media: 10,74 años).

Los pacientes se clasificaron de acuerdo con los criterios de la IHS de 2004 en: MC, 14 $(51,8 \%)$; CTC, 6 (22,2\%); CPDN, 4 (14,8\%); no clasificable, $3(11,1 \%)$

La localización de la cefalea se describió como localizada y bilateral en 22 pacientes (frontal en 16, frontotemporal en tres, biparietal en tres) y holocraneal en cuatro. Dos pacientes presentaban cefalea hemicraneal, en un caso asociada a cefalea holocraneal y en el otro a una cefalea frontal. Únicamente en este paciente la cefalea era estrictamente unilateral (frontal izquierda).

En 23 pacientes existía una historia familiar de cefaleas $(73,9 \%)$, y en 17 de ellos la madre era uno de los familiares.

Intentamos determinar si existían dos tipos de cefaleas o si se producía una cefalea con intensidad claramente variable. Después, intentamos correlacionar estas características con la producción de náuseas, vómitos, fonofobia y fotofobia e inhibición de la actividad física, así como con la clasificación del tipo de cefalea.

Los pacientes tuvieron dificultad para responder a la cuestión de si sufrían dos tipos de cefalea por el tipo de dolor, pero rápidamente respondieron a la cuestión de si había una significativa variación de la intensidad.

En 17 de los pacientes había una intensidad variable (14 clasificados como MC y dos como CPDN; dos pacientes no eran clasificables). Los pacientes clasificados como CPDN reconocían la variación de la intensidad entre leve y moderada, mientras que los pacientes con MC consideraban su cefalea como moderada e intensa. Todos los pacientes clasificados como CTC y dos como CPDN no reconocían la fluctuación de la intensidad en la cefalea (Tabla I).

En 18 pacientes se confirmó un consumo frecuente de analgésicos, bien durante la progresión de la cefalea intermitente hacia cefalea crónica, bien durante el período de cronicidad. 
La CCD en niños y adolescentes se reconoce como rara. Abu-Arafeh et al [2], en un estudio de la población, identificaron únicamente un $0,2 \%$ de niños con este diagnóstico. También es improbable que un niño o adolescente con un cuadro clínico de cefalea casi diaria no haya acudido a un centro con experiencia en cefaleas. Nuestro estudio no hace referencia a una población; sin embargo, señalamos que apenas 27 de los 397 niños $(6,25 \%)$ padecían cefaleas tipo CCD.

La edad media de nuestros pacientes es de 10,74 años, y el $69,4 \%$ era de sexo femenino, lo cual es muy parecido a los que se ha verificado en casuísticas mayores publicadas con anterioridad.

Con el empleo de la actual clasificación de la IHS, 14 pacientes presentaban MC (51,8\%), 6 CTC $(22,2 \%), 4$ CPDN $(14,8 \%)$ y 3 no eran clasificables.

Las casuísticas de CCD en adultos con la utilización de la clasificación de Silberstein revisada hacen referencia a un porcentaje más elevado de MC (aproximadamente un 78\%) e inferior de CTC (15\%), y se consideran raras la CPDN y la HC. Tres trabajos sobre niños y adolescentes obtuvieron una frecuencia más elevada de la cefalea crónica clasificable como CPDN en comparación con estudios efectuados en adultos, aunque el estudio de Esposito et al [9] no haya clasificado ningún caso.

En nuestros pacientes también se clasificaron cuatro casos catalogables como CPDN En todos estos casos, la cefalea era moderada o leve y dos casos $(50 \%)$ describían una variación de la intensidad. En todos, la cefalea era bilateral, frontal o frontotemporal, y ninguno presentó náuseas, vómitos o fotofobia.

Un estudio sobre factores precipitantes de CPDN [12] identificó infecciones víricas en un $40 \%$; pero, en nuestros casos no conseguimos identificar ninguna infección precedente.

Los resultados de los estudios sobre CCD en niños [13] son dispares entre sí, probablemente debido a la falta de uniformidad de los criterios de diagnóstico. La principal discrepancia es resultado de no incluir una categoría de 'cefalea comórbida tensional y migraña'.

El análisis de nuestros casos mostró un número significativo de niños con una fluctuación de la intensidad de la cefalea claramente identificable; pero estos pacientes dudaban a la hora de admitir dos tipos de distintos de cefalea (pulsátil o constrictiva o sensación de peso); además, la descripción del tipo de cefalea era considerablemente imprecisa y poco útil para el diagnóstico.

Excepto dos pacientes clasificables como CPDN -que mencionaban fluctuación de la intensidad entre leve y moderada- y uno con una cefalea no clasificada, todos los demás presentaban una elevada incidencia de la náuseas, fonofobia y, sobre todo, fotofobia e inhibición de la actividad.

Pensamos que pueden existir, por lo menos, dos elementos de dificultad en la clasificación de la CCD en niños: la menor capacidad discriminativa de los niños en cuanto al tipo de dolor y otro factor, intrínseco y posiblemente no relacionado con la edad, resultante de la modificación de las características del dolor con la evolución hacia la cronicidad, que pue- de ser consecuencia del hecho de que el CTC y MC comparten los mismos mecanismos centrales de producción del dolor.

En el trabajo de Hershey et al [7], la duración de la cefalea se consideró como el criterio más evidente de separación de los tres grupos: cefalea frecuente, diaria intermitente y diaria continua. Sin embargo, incluso en este mismo grupo las cefaleas todavía presentaban características migrañosas (náuseas, 48,7\%; fonofobia y fotofobia, respectivamente, en un $64,1 \%$ ). Este trabajo no identificó como distintiva la intensidad de la cefalea.

Aunque nos encontremos limitados en la interpretación por un pequeño número de casos, nos parece que el elemento de la historia clínica que los niños y adolescentes mencionan con mayor firmeza es la presencia de la fluctuación de la intensidad y que este elemento se correlaciona con características migrañosas de la cefalea. Por otro lado, no nos parece consistente la respuesta a la pregunta: ‘coexisten dos tipos de cefaleas?', ya que un gran número de pacientes tiene dificultad para precisar el tipo de cefalea, en particular los preadolescentes.

\section{J.P. Vieira, A.C. Ferreira, E. Veiga}

Aceptado tras revisión externa: 27.06.05.

Servicio de Neurología. Hospital de Dona Estefânia. Lisboa, Portugal.

Correspondencia: Dr. José Pedro Vieira. Serviço de Neurologia. Hospital de Dona Estefânia. Rua Jacinta Marto. 1159-045 Lisboa, Portugal. Fax +21312 69 63. E-mail: josepedrovieira@hotmail.com

\section{BIBLIOGRAFÍA}

1. Castillo J, Muñoz P, Guitera V, Pascual J. Epidemiology of chronic daily headache in the general population. Headache 1999; 39: 190-6

2. Abu-Arafeh I, Russel G. Prevalence of headache and migraine in schoolchildren. BMJ 1994; 309: 765-9.

3. Sillanpää M, Piekkala P, Kero P. Prevalence of headache at preschool age in an unselected child population. Cephalalgia 1991; 11: 239-42.

4. Silberstein SD, Lipton RB, Sliwinski M. Classification of daily and near-daily headaches: field trial of revised IHS criteria. Neurology 1996; 47: 871-5.

5. Gladstein J, Holden W. Chronic daily headache in children an adolescents: a 2-year prospective study. Headache 1996; 36: 349-51.

6. Abu-Arafeh I. Chronic tension type headache in children and adolescents. Cephalagia 2001; 21: 830-6.

7. Hershey AD, Powers SW, Bentti AL, LeCates S, De Grauw TJ. Characterization of chronic daily headaches in children in a multidisciplinary headache center. Neurology 2001; 56: 1032-7.

8. Koenig MA, Gladstein J, McCarter RJ, Hershey AD, Wasievski W. Chronic daily headache in children and adolescents presenting to tertiary headache clinics. Headache 2002; 42: 491-500.

9. Esposito SB, Gherpelli JLD. Chronic daily headaches in children ad adolescents: a study of clinical characteristics. Cephalalgia 2004; 24: 476-82.
10. Moore AJ, Shevell M. Chronic daily headaches in pediatric neurology practice. $\mathrm{J}$ Child Neurol 2004; 19: 925-9.

11. Santoni JR, Santoni-Williams CJ. Headache and painful lymphadenopathy in extracranial or systemic infection: etiology of new daily persistent headaches. Intern Med 1993; 32: 530-2.

12. Díaz-Mitoma F, Vanast WJ, Tyrrell DL. Increased frequency of Epstein-Barr virus excretion in patients with new daily persistent headaches. Lancet 1987; 1: 411-5.

13. Mack KJ. What incites new daily persistent headache in children? Pediatr Neurol 2004; 31: $122-5$.

\section{Parálisis del nervio radial por exposición a plomo}

El plomo ha estado presente en el organismo humano desde tiempos remotos; fue uno de los primeros metales conocidos por el hombre [1], y es, quizás, el de mayor interés toxicológico. Los egipcios utilizaban el plomo y conocían muy bien el saturnismo, que fue explícitamente descrito hace más de 2.000 años por el médico y poeta griego Nicandro. En el siglo I, Dioscórides describió el cólico y la parálisis saturnina [2]. Bernardino Ramazzini, médico italiano, considerado el padre de la medicina del trabajo, describe alrededor de 1700 , en su libro De morbis artificium diatriba, las enfermedades que padecían los ceramistas y los pintores. El tratado de Tanquerel des Planches Traité des maladies du plomb ou saturnisme (1839) recoge la descripción clínica de más de 1.000 casos de intoxicación, y sus datos siguen teniendo vigencia en nuestros días [3].

En los últimos años se ha constatado un aumento del número de estudios sobre efectos del plomo en una población general expuesta a bajas concentraciones. La mayor cantidad de plomo presente en el aire procede de la actividad humana. Aproximadamente el $90 \%$ presente en la atmósfera de las ciudades proviene de procesos industriales y de la combustión de gasolina.

Las actividades de mayor riesgo son aquellas en las que se calienta el plomo metálico o inorgánico y se forman aerosoles y humos en grandes cantidades.

Los valores límite de exposición no deben superarse; por encima de éstos, debe alejarse al trabajador del puesto habitual y de la exposición en general.

Se considera trabajador expuesto al riesgo de plomo a todo aquel que tiene plomo en sangre y que durante más de 30 días al año ejerce su actividad laboral ( 8 horas diarias y 40 semanales) en un ambiente con una concentración de plomo $\geq 40 \mu \mathrm{g} / \mathrm{m}^{3}$ [4].

Después de cierto tiempo de exposición, el plomo puede acumularse en el organismo y afectar principalmente a cuatro órganos o sistemas: hematopoyético, gastrointestinal, renal y nervioso, y especialmente a este último.

Describimos las características clínicas, bioquímicas y neurofisilógicas de un caso diagnosticado de parálisis del nervio radial bilateral por exposición a plomo. 